Running Head: NEURAL SUBSTRATES OF LINGUISTIC PROSODY

NEURAL SUBSTRATES OF LINGUISTIC PROSODY: EVIDENCE FROM

SYNTACTIC DISAMBIGUATION IN THE PRODUCTIONS OF BRAIN-DAMAGED

\title{
PATIENTS
}

Amee P. Shah, Shari R. Baum, \& Veena D. Dwivedi

School of Communication Sciences and Disorders, McGill University, Montreal, Canada

Author to whom correspondence should be addressed:

Amee P. Shah

Department of Speech and Hearing

Cleveland State University

2121 Euclid Avenue, MC 430

Cleveland, OH 44115-2214

Tel: 216-687-6988; Fax: 216-687-6993

a.shah101@csuohio.edu 


\begin{abstract}
The present investigation focussed on the neural substrates underlying linguistic distinctions that are signalled by prosodic cues. A production experiment was conducted to examine the ability of left- (LHD) and right- (RHD) hemisphere damaged patients and normal controls to use temporal and fundamental frequency cues to disambiguate sentences which include one or more Intonational Phrase level (IPh) prosodic boundaries. Acoustic analyses of subjects' productions of three sentence-types - parentheticals, appositives, and tags - showed that LHD speakers, compared to RHD and normal controls, exhibited impairments in the control of temporal parameters signalling phrase boundaries, including inconsistent patterns of pre-boundary lengthening and longer-thannormal pause durations in non-boundary positions. Somewhat surprisingly, a perception test presented to a group of normal native listeners showed listeners experienced greatest difficulty in identifying the presence or absence of boundaries in the productions of the RHD speakers. The findings support a cue lateralization hypothesis in which prosodic domain plays an important role.
\end{abstract}

Keywords: prosodic control, acoustic measures, duration, F0, syntactic disambiguation, brain-damage, lateralization. 


\section{NEURAL SUBSTRATES OF LINGUISTIC PROSODY: EVIDENCE FROM \\ SYNTACTIC DISAMBIGUATION IN THE PRODUCTIONS OF BRAIN-DAMAGED PATIENTS}

\section{Introduction}

The contributions of the left and right cerebral hemispheres to the processing of speech prosody have been the object of a great deal of study in recent years. Despite the increased interest in this issue, the neural substrates for prosodic processing remain unresolved. Several competing hypotheses concerning the lateralization of prosodic processing exist—most based largely on data from perception or comprehension studies (see Baum \& Pell, 1999 for review). In particular, the functional lateralization hypothesis (Van Lancker, 1980) proposes that hemispheric involvement varies with the functional significance of the prosodic stimulus or task, with linguistic prosody processed within the left hemisphere (LH) and affective prosody processed within the right hemisphere $(\mathrm{RH})$. A second hypothesis, the cue dependent hypothesis (Van Lancker \& Sidtis, 1992), argues that temporal prosodic cues are left-hemisphere lateralized, whereas spectral cues are right-hemisphere lateralized. Quite recently, several new but related proposals concerning speech processing, in general, have been put forth, which emphasize the duration of the temporal integration windows required for signal processing as determining factors in lateralization (e.g., Gandour et al., 2003; Poeppel, 2003). Obviously, the different hypotheses make quite different predictions regarding prosodic lateralization; to date, however, no single theory has garnered consistent support. These perceptually-based theories — when applied to the realm of prosody production — have yielded even more inconsistent findings. 
For example, looking only at linguistic prosody, numerous investigators have reported impairments in the control of temporal parameters of speech prosody in lefthemisphere-damaged (LHD) individuals (e.g., Baum \& Pell, 1997; Baum et al., 2001; Danly \& Shapiro, 1982; Danly et al., 1983; Gandour et al., 1993; 1994; 2000; Schirmer et al., 2001), but relatively spared control of timing in individuals with right hemisphere damage (RHD) (Baum \& Boyczuk, 1999; Baum \& Pell, 1997; Gandour et al, 1994; Schirmer et al., 2001; Walker et al., 2004). However, investigations of the control of F0 in brain-damaged speakers have yielded mixed results, sometimes demonstrating deficits on the part of LHD speakers (e.g., Cooper et al., 1984; Danly \& Shapiro, 1982; Danly et al., 1983; Ryalls, 1982) and sometimes showing deficits for RHD speakers (e.g., Baum \& Pell, 1997; Behrens, 1989; Bradvik et al., 1991; Bryan, 1989; Shapiro \& Danly, 1985; Weintraub et al., 1981). (See also Mayer and colleagues, 1999; 2002 for some interesting fMRI findings with respect to prosody production.)

Whereas the majority of studies of the production (and perception; see e.g., Heilman et al., 1984; Pell \& Baum, 1997a; Weintraub et al., 1981) of linguistic prosody in brain-damaged patients have concentrated on global acoustic cues to sentence type (i.e., declarative vs. interrogative vs. imperative), there has recently been a surge of interest in more subtle, but extremely important linguistic distinctions that are signalled by prosodic cues in both normal speech production (e.g., Beach, 1991; Grabe \& Warren, 1995; Nagel et al., 1994; Price et al., 1991), as well as in production by brain-damaged patients (e.g., Baum et al., 1997; Grela \& Gandour, 1998;1999; Schirmer et al., 2001; Walker et al., 2004; see also Grosjean \& Hirt, 1996; Marslen-Wilson et al,.1992; Nagel et al., 1994; Shapiro \& Nagel, 1995; Walker et al., 2001; 2004 for data on perception). Of 
particular relevance to the present investigation are a number of investigations that have examined the ability of brain-damaged patients to mark syntactic boundaries using prosodic cues. In one of the earlier studies to explore prosodic cues to syntactic disambiguation in LHD and RHD patients, Baum et al. (1997) investigated the ability of such patients to mark simple phrasal groupings in a conjoined string ('pink and black and green'; see also Katz et al., 1996). Results revealed that both the LHD and RHD speakers produced relatively normal patterns of pre-boundary lengthening and increased pause durations at boundary positions; however, the increased durations were not as consistent as in non-brain-damaged speakers. Moreover, perceptual analyses indicated that the boundary cues produced by the brain-damaged speakers were less perceptible than those produced by the normal speakers. None of the three speaker groups produced consistent F0 cues signalling these simple phrase boundaries.

In a further investigation of prosodic cues to disambiguation, Baum et al. (2001) explored three syntactically ambiguous sentence types: adjectival scope ambiguities (Lehiste, 1973), prepositional phrase attachment ambiguities (Lehiste et al., 1976) and noun phrase attachment ambiguities (Beach, 1988; 1991; Shapiro \& Nagel, 1995; Stirling \& Wales, 1996; Warren, 1985). As in the previous study, acoustic analyses suggested relatively spared abilities to signal the syntactic constituent structure using temporal cues in both LHD and RHD participants. Again, analyses of the F0 patterns yielded few of the expected cues in any speaker group (see also Albritton et al., 1996). Schirmer et al. (2001) have also found largely unimpaired prosody production abilities in both LHD and RHD speakers in a task requiring the shifting of stress across sentence positions to indicate focus. Nonetheless, as in previous studies (e.g., Baum et al., 1997; Walker et al., 
2004), listeners exhibited greater difficulty in identifying the position of contrastive emphasis in productions of the brain-damaged individuals relative to control subjects. Similarly, in an examination of the speech of RHD patients compared to non-braindamaged speakers, Walker and colleagues (2004) reported normal prosodic cues to the sentence-question distinction, lexical stress distinctions, and the cueing of syntactic boundaries (see also Pell, 1999; but cf. Hird \& Kirsner, 1993; Shapiro \& Danly, 1985; Weintraub et al., 1981); however, listeners were less able to accurately perceive the productions of the RHD speakers for the syntactic disambiguation stimuli. Taken together, these findings suggest that there may be acoustic differences present that are not being captured by the analyses conducted to date.

It is clear that there is not yet a consensus regarding the ability of speakers to successfully utilize prosodic cues to signal linguistic structural differences subsequent to LHD or RHD. Moreover, given recent hypotheses concerning the neural bases of prosodic processing — in particular, Gandour et al.'s (2003) contention that the linguistic prosodic domain plays an important role in determining hemispheric involvement (see also Behrens, 1989; Baum \& Dwivedi, 2003), further examination of the prosody production abilities of LHD and RHD patients is warranted. The current investigation explores three types of syntactic constructions - parentheticals, appositives and tags. All three of these constructions contain phrases that can alternatively be syntactically and semantically independent of the sentences they modify (i.e., unintegrated) or dependent (i.e., integrated) (Emonds, 1979). This dependency (or lack thereof) is reflected in the presence or absence of a prosodic boundary (e.g., Intonational Phrase [IPh]; Nespor \& Vogel, 1986; Selkirk, 1984) within the sentence. For example, prosodic cues alone serve 
to indicate the appropriate interpretation of sentences with parenthetical versus integrated phrases (e.g., in the sentence "Mel knew by the way you were driving," it is unclear (without prosodic cues, or punctuation in written language) whether "by the way" is a parenthetical remark or is semantically relevant to the interpretation of the entire sentence-i.e., the sentence could mean 'Mel knew that you were driving' or 'Mel knew something about you because of the manner in which you were driving'). These structures are of particular interest because they include major prosodic breaks (IPhs) within sentences; the majority of previous investigations have focused on less major break indices (e.g., Phonological Phrases [PPhs]) within sentences.

In normal speakers, for these types of structural ambiguities, pre-boundary words and phones are increased in duration, as are pauses at the boundaries (Price et al.,1991). Further, intonation analyses show the presence of IPh boundary tones (i.e., fall-rise or continuation rise patterns) at major syntactic boundaries (Price et al., 1991). Price and colleagues (1991) found that these cues were perceptible, in that listeners were able to reliably disambiguate the segmentally identical sentences using the prosodic cues alone. Based on these findings, we adapted a subset of the sentence types used by Price and colleagues (1991) for investigation of the prosody production abilities of LHD and RHD speakers.

As prosodic boundaries at the sentence level subserve linguistic functions and are primarily marked by temporal cues (syllable lengthening, pause insertion), both the functional lateralization hypothesis (Van Lancker, 1980) and traditional cue-dependent theories (Liegeois-Chauvel et al., 1999; Zatorre et al., 1992) predict more difficulties in LHD than RHD patients. In contrast, recent theories emphasizing that the duration of 
temporal integration windows determines lateralization of processing (Gandour et al., 2003; Poeppel, 2003), predict difficulties at the sentence level for RHD rather than LHD patients.

\section{METHODS: ACOUSTIC STUDY}

\section{Subjects}

Twenty-one subjects participated in the experiment: 6 LHD nonfluent aphasic patients, 7 RHD patients, and 8 age-matched non-brain-damaged control subjects. All were native English speakers with hearing within normal limits (PTA bilaterally $<30 \mathrm{~dB}$ at frequencies of $500 \mathrm{~Hz}, 1 \mathrm{kHz}$, and $2 \mathrm{kHz}$ ). As indicated from their medical records, the brain-damaged patients had all suffered a single, unilateral CVA, and at the time of participation in this study, they were all at least 3 years post onset of the CVA. Additional details regarding the background information of the three subject groups are provided in Table $1^{1}$.

All brain-damaged subjects underwent a battery of screening tests including sections of the Psycholinguistic Assessment of Language (Caplan, 1992), Mini Mental State Examination (Folstein et al., 1975), the Behavioural Inattention Test (Wilson et al., 1997), and the Discourse Comprehension Test (Brookshire \& Nicholas 1993) to ensure adequate reading skills, auditory comprehension, auditory memory, discourse comprehension, and to rule out visual neglect. In addition, the LHD patients were administered sections from the Boston Diagnostic Aphasia Examination (Goodglass \&

\footnotetext{
${ }^{1}$ Whereas it would have been preferable for all participants to have more homogeneous sites of lesionand, in particular, to have excluded those individuals with purely subcortical lesions - this was not feasible given the limited pool of patients available to us. It was seen, however, that the data from those patients with subcortical lesions were within 2 standard deviations of the group averages, thus did not differ substantively from the rest of the group.
} 
Kaplan, 1983) and the Boston Naming Test (Kaplan et al., 1983) to characterize their difficulties with verbal expression. Only those individuals who exhibited deficits typical of nonfluent aphasia ${ }^{2}$, and were free from dysarthria (based on speech-language pathology reports), were included in the study. Correspondingly, the RHD patients were administered additional screening tests to tap problems typically associated with right hemisphere brain damage, including difficulties with drawing inferences and interpreting figurative language, as well as difficulties with perception of emotional prosody (sections derived from the Test of Language Competence-Expanded Edition, Wiig \& Secord, 1988). Only those individuals with impairments on at least one of these tests (again, free from dysarthria) were included as participants.

\section{Stimuli}

Stimuli were comprised of sentence pairs, modelled after Price et al. (1991), that were segmentally matched (same string of phonemes and/or words) but syntactically different, so that only prosodic cues could disambiguate them. Each of eight sentences of three types appeared in two versions — integrated and non-integrated — for a total of 48 stimuli. The non-integrated versions included parentheticals, appositives and tag constructions; the integrated versions of these sentence types were identical, but included no syntactic/prosodic boundaries. For each target sentence, a context was created to bias the intended reading (i.e. integrated or non-integrated). Table 2 provides examples of

\footnotetext{
${ }^{2}$ We consider the articulatory disturbance characteristic of many individuals with nonfluent aphasia equivalent to 'apraxia of speech' (AOS). There is much debate as to the concept of AOS which is beyond the scope of the present investigation. Patients were not screened for AOS, but none was reported in their records. Further, it is not critical for the arguments in the present study, whether articulatory implementation deficits are 'linguistic' or 'motoric' in nature.
} 
each of the three sentence types, in their integrated and non-integrated versions, along with the associated biasing contexts.

\section{Procedure}

Testing was conducted over two days separated by at least one week to avoid exaggeration of contrasts in disambiguation. The stimulus corpus was counterbalanced for presentation, such that on day one, half the speakers were presented with List 1 (comprising 12 non-integrated sentences and 12 integrated ones), and the other half of the speakers with List 2 (the 24 remaining non-integrated and integrated sentences). The list order was reversed on day two.

Subjects were asked to silently read the biasing sentence, and then read the target sentence aloud, trying to convey the meaning they interpreted from the preceding (biasing) sentence. The productions were recorded on a Sony DAT recorder (Model TCD-D100) with a head-mounted directional microphone (Model AKG 420), and then digitized with 12-bit resolution at a sampling rate of $10 \mathrm{kHz}$ using the Bliss speech analysis system (Mertus, 1989) for acoustic analysis.

\section{Acoustic Analyses}

The Multispeech waveform editing software, Model 3700, version 2.3 (Kay Elemetrics, 1999) and the Bliss speech analysis system were used to perform the acoustic analyses. Acoustic analyses involved temporal and fundamental frequency (F0) measures of elements within each sentence type. Multispeech displays of waveforms and wideband spectrograms were used in combination to make decisions regarding segment boundaries, 
as well as in computing temporal measures. F0 measures for target syllables (as detailed below) were estimated from an average of three periodic pulses near the vocalic midpoint, as determined from the waveform display. To examine F0 changes at the boundaries, pre-boundary/post-boundary F0 ratios were calculated. Temporal analyses included overall sentence durations, as well as pre-boundary word durations and pause durations at the (potential) boundaries. Whereas some stimuli had two potential boundaries, the majority included only one; therefore measures were made on the first potential boundary in each sentence. For example, in the parenthetical exemplar from Table 2, the durations of segments measured were those of "him”, "think" and the pause after "him"; in the Tag exemplar, the measured durations comprised the segments "leave”, "would he/ Woody” and the pause between them. To control for speech rate differences across participants, word and pause durations were expressed as a proportion of the full sentence duration.

\section{METHODS: PERCEPTION TEST}

\section{Subjects}

A perception test was also constructed for presentation to a group of ten non-braindamaged native-English-speaking, phonetically-trained listeners (mean age: 21 years) to determine whether the contrasts produced by the various speaker groups were perceptible. 


\section{Stimuli}

Four sentence pairs were selected at random from each of the three sentence types for each speaker in each group, for a total of $504^{3}$ stimuli. These stimuli were split into two lists, which were randomized for presentation to the listeners.

\section{Procedure}

Testing was conducted over two sessions separated by at least one week. Each listening session lasted approximately 30 minutes. Stimuli were presented in a quiet room over closed headphones via the Bliss AV Runner program at an ISI of 4 seconds. Listeners were given an answer-sheet containing all the stimulus sentences in their integrated and non-integrated versions, including disambiguating punctuation. For each target sentence presented, listeners were asked to determine which version of the two written sentences they heard.

\section{RESULTS}

Analyses were conducted for each sentence type and each acoustic measure separately. Group (NC, LHD, RHD) x Boundary (integrated, non-integrated) analyses of variance (ANOVAs) were conducted on pre-boundary word proportional durations, pause proportional durations and F0 ratios.

\section{Temporal Analyses}

For the Parenthetical sentence type, mean pre-boundary proportional durations are displayed in Fig. 1a. As illustrated in the figure, all groups showed a tendency to exhibit pre-boundary lengthening. Statistical analyses revealed a main effect of Group [F

\footnotetext{
3 Three of these stimuli had to be discarded due to speaker error.
} 
$(2,18)=7.813, \mathrm{p}<.01]$ and an interaction of Group $x$ Boundary condition $[F(2,18)=$ 3.713, $\mathrm{p}<.05]$. Simple effects analyses revealed that the Boundary effect only reached significance for the NC participants [F (1,7) $=20.791, \mathrm{p}<.01]$. For the Appositive sentence type (Fig. 1b), all groups again showed the expected pre-boundary lengthening pattern. The ANOVA revealed significant main effects for Group [F $(2,18)=8.056, p<.01]$ and Boundary [F $(1,18)=14.367, \mathrm{p}<.01]$. The Group x Boundary interaction was significant $[\mathrm{F}(2,18)=6.167, \mathrm{p}<.01]$, and simple effects analyses showed that the Boundary effect reached significance for the NC group [F $(1,7)=19.925, \mathrm{p}<.01]$ and the RHD group [F $(1,7)=7.107, \mathrm{p}<.05]$. In the Tag sentences, Fig. 1c illustrates a similar pattern across the groups. The ANOVA yielded main effects for Group $[F(2,18)=7.360$, $\mathrm{p}<.01]$ and Boundary $[\mathrm{F}(1,18)=8.286, \mathrm{p}<.05]$. Again, the interaction between Group and Boundary was significant $[\mathrm{F}(2,18)=5.88, \mathrm{p}<.05]$, and simple effects analyses revealed that the Boundary effect was significant in the NC group alone [F(1,7)=13.098, $\mathrm{p}<.01]$.

Fig. 2(a-c) displays mean proportional pause durations for all three groups for all sentence types. For the Parentheticals (Fig. 2a), all groups showed the expected tendency to produce longer pauses at syntactic boundaries, as confirmed by the ANOVA, which revealed significant main effects of Group $[\mathrm{F}(2,18)=7.902, \mathrm{p}<.01]$ and Boundary $[\mathrm{F}$ $(1,18)=14.690, \mathrm{p}<.01]$. If each group is analyzed separately, the effect of Boundary is significant only in the LHD group [F $(1,5)=15.271, \mathrm{p}<.05]$. For the Appositives (Fig. 2b), a similar pattern emerged, with a significant main effect of Boundary alone $[F(1,18)$ $=38.908, \mathrm{p}<.01]$, and simple effects analyses showed the effect of Boundary to be significant in all groups, indicating that all groups showed a similar pattern. Similarly, for 
the Tags (Fig. 2c), only a significant main effect of Boundary $[\mathrm{F}(1,18)=12.420, \mathrm{p}<.01]$ was found.

Interestingly, in the integrated phrase conditions-across all sentence types-the LHD patients always produced longer pause durations than either the NC or RHD participants. In other words, the LHD patients appeared to be producing pauses in inappropriate conditions, i.e., in non-pause expected (integrated) versions of the sentences.

\section{F0 Analyses}

Mean pre-boundary/post-boundary F0 ratios were compared across the integrated and non-integrated conditions in each of the three sentence-types, across the three subject groups. As illustrated in Fig. 3 a-c, the expected pattern of fall-rise in F0 from preboundary to post-boundary words was observed consistently in only the RHD group. Group (NC, LHD, RHD) x Boundary (integrated, non-integrated) ANOVAs performed on the F0 ratios for each of the three sentence types failed to reveal any significant main effects or interactions.

\section{Perceptual Analyses.}

For each listener, median percent errors collapsed across the speakers in each subject group were computed for each sentence type and syntactic break condition. Fig. 4 shows percent errors for the subject group, averaged over the listeners. Across all sentence types, listeners exhibited greater difficulty identifying the presence or absence of a boundary in the speech of the brain-damaged patients relative to the NC speakers. Interestingly, perception of the distinctions as signalled by the LHD speakers was 
somewhat more accurate than for the RHD speakers overall. It is noteworthy that listeners had greater difficulty with the integrated versions of all sentence types produced by the LHD speakers, likely due to the inappropriate pausing noted above. Listeners produced relatively more errors in the parenthetical sentence type across all groups. A Group x Boundary condition (integrated/non-integrated) repeated measures ANOVA was conducted on the median percent errors collapsed across the listeners. For the Appositive sentence type, significant effects of Group [F $(2,6)=10.690, \mathrm{p}<.05]$ and Boundary condition $[\mathrm{F}(1,7)=0.026, \mathrm{p}<.01$, as well as an interaction of Group $\mathrm{x}$ Condition [F $(2,6]=8.550, \mathrm{p}<.018]$ were found. Post-hoc tests showed an effect of Boundary condition in the RHD group only. For the Parenthetical sentence type, a main effect of Group [F $(2,6)=6.852, \mathrm{p}<0.028]$ and an interaction of Group and Boundary condition [F $(2,6)=20.024, \mathrm{p}<.05]$ were found. Again, post-hoc analyses showed the effect of condition was significant in the RHD group only. For the Tag sentence type, a main effect of Boundary condition $[F(1,7)=45.97, \mathrm{p}<.01]$ and an interaction of Boundary condition and Group [F (2,6)=11.93, $\mathrm{p}<.01]$ were noted. Post-hoc analyses showed the Boundary effect was significant only in the LHD group.

In order to identify any individual speakers whose productions may have been particularly difficult to perceive, the perceptual data were also analyzed by speaker, collapsed across listeners. As illustrated in Table 3, results showed that each of the individual speakers largely conformed with their respective group pattern with three clear exceptions - the integrated Parentheticals produced by LHD 3 and NC 7 and,the nonintegrated Appositives produced by RHD 4. There were no obvious characteristics that differentiated these individuals from the remainder of their group. 


\section{DISCUSSION}

The findings of the present investigation are consistent with those of several recent experiments that have shown relatively normal patterns of acoustic cues to sentence-level prosodic distinctions as produced by both LHD and RHD patients (e.g., Baum et al., 2001; Schirmer et al., 2001; Walker et al., 2004). Despite the relatively preserved patterns, individuals with LHD in particular, displayed less consistent use of temporal parameters in signaling the intonational phrase boundaries (and hence the syntactic distinctions) in the present investigation. For example, while all three groups of participants exhibited pre-boundary lengthening and increased pause durations at boundaries, the pre-boundary durational differences always failed to reach significance for the LHD patient group. Further, and again in keeping with previous findings, the LHD patients produced integrated phrases with longer-than-normal pauses, suggesting that these speakers were pausing in inappropriate sentence positions (see e.g., Kent \& Rosenbek, 1982; 1983). This type of speech production pattern is, of course, not unexpected, and in fact reflects one of the reasons for the designation 'nonfluent' aphasia.

With respect to analyses of F0, as in numerous other investigations (e.g., Baum et al., 1997; 2001; see also Albritton et al., 1996), none of the speaker groups consistently demonstrated the expected fall-rise patterns at major boundaries. Somewhat contrary to predictions based on cue lateralization hypotheses (e.g., Gandour et al., 2003; Poeppel, 2003; Van Lancker \& Sidtis, 1992), the speakers who produced the most consistent pattern of boundary tones were the individuals with RHD, although it should be borne in mind that none of the F0 comparisons reached statistical significance for any group. These results suggest either that normal speakers do not utilize F0 in a consistent manner 
to mark the type of syntactic boundaries examined in the present study (Albritton et al., 1996; Stirling \& Wales, 1996; Walker et al., 2004) or that the specific measures used do not adequately reflect the actual distinguishing features produced (e.g., Baum et al., 2001; but cf. Blasko \& Hall, 1998; Ladd, 1988; Price et al., 1991; Warren, 1985) ${ }^{4}$.

The results of the perceptual experiment indicate that, in general, listeners were able to perceive the cues used by all speaker groups to mark the syntactic boundaries. Nonetheless, across the sentence types, perception was substantially less accurate for the productions of the RHD speakers (25\% errors) relative to the LHD speakers (19\% errors), whose productions were, in turn, harder to identify than those of the non-brain-damaged speakers (14\% errors). It is particularly interesting, as illustrated in Figure 4, that listeners generally had greater difficulty correctly identifying the integrated (compared to the non-integrated) phrases produced by the LHD participants. This is most likely due to the inappropriate pauses produced by the LHD speakers. For the other speaker groups, error rates were less clearly distributed in one condition over the other. It is also interesting to note the very high error rate for the non-integrated parenthetical stimuli, as produced by the RHD speakers. The parenthetical phrases, in most cases, may be considered to constitute a form of 'formulaic language' which may be particularly vulnerable in individuals with RHD (e.g., Van Lancker, 2004) ${ }^{5}$, thus providing a possible alternative account of the somewhat surprising difficulty experienced by listeners with these sentences. It has also been proposed that processing of proper nouns may be more impaired subsequent to RHD (Van Lancker \& Ohnesorge, 2002), whereas common nouns may be more affected by LHD. Upon close inspection, it is evident that more

\footnotetext{
${ }^{4}$ It is, of course, also possible that the results failed to reach statistical significance due to the small number of subjects in each group.

${ }^{5}$ We are grateful to an anonymous reviewer for pointing this out.
} 
proper nouns appear in the non-integrated versions of the appositive stimuli and the integrated versions of the tag stimuli-precisely those conditions in which perceptual errors were greatest for the RHD speakers' productions. While there are an insufficient number of stimuli of the different types to fully address this possibility, it remains an intriguing issue that should be addressed in future investigations.

Given the absence of clear F0 cues in the stimuli, the overall findings suggest that temporal parameters may be sufficient to signal intonational boundaries of the type examined here (see also Shapiro \& Nagel, 1995). Despite the relatively normal durational patterns produced by the RHD speakers, perception of the contrasts as produced by these speakers was much less accurate than for the other groups; these findings suggest that the RHD speakers were less able to adequately signal IPh boundaries, consistent with hypotheses that highlight the importance of the prosodic domain in determining hemispheric lateralization of prosodic processing (e.g., Baum \& Dwivedi, 2003; Behrens, 1989; Gandour et al., 2003; Schirmer et al., 2001). The relatively mild impairments in temporal control exhibited by the LHD participants are consistent with the vast majority of previous investigations supporting a LH lateralization for the control of speech timing (e.g., Efron, 1963; Smith, 1980; see Blumstein, 1998 for review). Taken together, the findings are largely supportive of a cue lateralization hypothesis in which prosodic domain plays an important role (e.g., Gandour et al., 2003; Schirmer et al., 2001; see also Van Lancker \& Sidtis, 1992). 


\section{REFERENCES}

Albritton, D.W., McKoon, G. \& Ratcliff, R. (1996). Reliability of prosodic cues for resolving syntactic ambiguity. Journal of Experimental Psychology: Learning, Memory, and Cognition, 22, 714-735.

Baum, S. R. \& Pell, M. D. (1997). Production of affective and linguistic prosody by brain-damaged patients. Aphasiology, 11, 177-198.

Baum, S. R. and Pell, M.D. (1999). The neural bases of prosody: insights from lesion studies and neuroimaging. Aphasiology, 13 (8), 581-608.

Baum, S.R., Pell, M.D., Leonard, C.L. \& Gordon, J.K. (1997). The ability of right- and left-hemisphere-damaged individuals to produce and interpret prosodic cues marking phrasal boundaries. Language and Speech, 40 (4), 313-330.

Baum, S. and Boyczuk, J. (1999). Speech timing subsequent to brain damage: Effects of utterance length and complexity. Brain and Language, 67, 30-45.

Baum, S., \& Dwivedi, V. (2003). Sensitivity to prosodic structure in left-and righthemisphere-damaged individuals. Brain and Language, 87, 278-289.

Baum, S., Pell, M., Leonard, C. \& Gordon, J. (2001). Using prosody to resolve temporary syntactic ambiguities in speech production: Acoustic data on brain-damaged speakers. Clinical Linguistics and Phonetics, 15, 441-456.

Beach, C. M. (1991). The interpretation of prosodic patterns at points of syntactic structure ambiguity: Evidence for cue trading relations. Journal of Memory and Language, 30, 644-663.

Beach, C. M. (1988). The influence of higher level linguistic information on production of duration and pitch patterns at syntactic boundaries. Journal of Acoustical 
Society of America, 75, p. S1.

Behrens, S. (1989). Characterizing sentence intonation in a right hemisphere-damaged population. Brain and Language, 37, 181-200.

Blasko, D. G. \& Hall, M. D. (1998). Influence of prosodic boundaries on comprehension of spoken English sentences. Perceptual and Motor Skills, 87, 3-18.

Blumstein (1998). Phonological aspects of aphasia. In M.T.Sarno (Ed.), Acquired Aphasia (Third Edition). San Diego: Academic Press.

Brådvik, B., Dravins, C., Holtås, S., Rosén, I., Ryding, E. \& Ingvar, D. (1991). Disturbances of speech prosody following right hemisphere infarcts. Acta Neurologica Scandinavica, 84, 114-126.

Brookshire, R.H., \& Nicholas, L.E. (1993). Discourse Comprehension Test. Minneapolis, Minnesota: BRK Publishers.

Bryan, K. (1989). Language prosody and the right hemisphere. Aphasiology, 3, 285-299.

Caplan, D. (1992). Language: Structure, processing and disorders. MIT Press, Cambridge, MA.

Cooper, W. E., Soares, C., Nicol, J. (1984). Clausal intonation after unilateral braindamage. Language and Speech, 27, 17-24.

Danly, M. and Shapiro, B. (1982). Speech prosody in Broca’s aphasia. Brain and Language, 16 (2), 171-190.

Danly, M., Cooper, W. E., Shapiro, B. (1983). Fundamental-frequency, language processing, and linguistic structure in Wernicke’s aphasia. Brain and Language, 19 (1): 1-24.

Efron, R. (1963). Temporal perception, aphasia and déjà vu. Brain, 86, 403-424. 
Emonds, J. (1979). Appositive Relatives Have No Properties. Linguistic Inquiry. 10, 211-43.

Folstein, M. F., Folstein, S. E., \& McHugh, P. R. (1975). Mini-mental state: A practical method for grading the cognitive state of patients for the clinician. Journal of Psychiatric Research, 12, 189-198.

Goodglass, H. \& Kaplan, E. (1983). The assessment of aphasia and related disorders. Lea and Febiger, Philadelphia, PA.

Gandour, J., Dechongkit, S., Ponglorpisit, S., \& Khunadorn, F. (1993). Intraword timing relations in Thai after unilateral brain-damage, Brain and Language, 45, 160-179.

Gandour, J., Dechongkit, S., Ponglorpisit, S. \& Khunadorn, F. (1994). Speech timing at the sentence level in Thai after unilateral brain damage. Brain and Language, 46, 419-438.

Gandour, J., Wong, D., Hsieh, L., Weinzapfel, B., Van Lancker, D., and Hutchins, G. D. (2000). A cross-linguistic PET study of tone perception. Journal of Cognitive Neuroscience, 12, 207-222.

Gandour, J., Dzemidzic, M., Wong, D., Lowe, M., Tong, Y., Hsieh, L., Satthamnuwong, N. \& Lurito, J. (2003). Temporal integration of speech prosody is shaped by language experience: An fMRI study. Brain and Language, 84, 318-336.

Grabe, E. \& Warren, P. (1995). Stress Shift: do speakers do it or do listeners use it? In B. Connell and A. Arvaniti (eds.), Papers in Laboratory Phonology IV. Phonology and Phonetic Evidence. Cambridge: CUP.

Grela, B., \& Gandour, J. (1999). Stress shift in aphasia: a multiple case study. Aphasiology, 13, 151-166. 
Grela, B., \& Gandour, J. (1998). Locus of functional impairment in the production of speech rhythm after brain damage: A preliminary study. Brain \& Language, 64, 361-376.

Grosjean, F., \& Hirt, C. (1996). Using prosody to predict the end of sentences in English and French: Normal and brain-damaged subjects. Language and Cognitive Processes, 11, 107-134.

Heilman, K. M., Bowers, D., Speedie, L., Coslett, H. B. (1984). Comprehension of affective and nonaffective prosody, Neurology, 34, 917-921.

Hird, K. \& Kirsner, K. (1993). Dysprosody following acquired neurogenic impairment. Brain and Language, 45, 46-60.

Kaplan, E, Goodglass, H. \&. Weintraub, S. (1983). Boston Naming Test. Philadelphia, PA: Lea and Febiger.

Katz, W., Beach, C., Jenouri, K., \& Verma, S. (1996). Duration and fundamental frequency correlates for phrase boundaries in productions by children and adults. Journal of the Acoustical Society of America, 99, 3179-3191.

Kay Elemetrics (1999). Multispeech Model 3700, Kay Elemetrics Corp., New Jersey.

Kent, R. D. \& Rosenbek, J. C. (1982). Prosodic disturbance and neurologic lesion. Brain and Language, 15, 259-291.

Kent, R. D. \& Rosenbek, J. C. (1983). Acoustic patterns of apraxia of speech. Journal of Speech and Hearing Research, 26, 231-249.

Ladd, D. R. (1988). Declination reset and the hierarchical organization of utterances. Journal of the Acoustical Society of America, 84, 530-544.

Lehiste, I. (1973). Phonetic disambiguation of syntactic ambiguity. Glossa, 7, 107-122. 
Lehiste, I., Olive, J. P., Streeter, L. A. (1976). Role of duration in disambiguating syntactically ambiguous sentences. Journal of the Acoustical Society of America, 60, 1199-1202.

Liegeois-Chauvel, C., de Graaf, J. B., Laguitton, V. \& Chauvel, P. (1999). Specialization of left auditory cortex for speech perception in man depends on temporal coding. Cerebral Cortex, 9, 484-496. [check)

Marslen-Wilson, W. D., Tyler, L. K., Warren, P., Grenier, P. \& Lee, C. S. (1992). Prosodic effects in minimal attachment. Quarterly Journal of Experimental Psychology, 45A, 73-87.

Mayer, J. (1999). Prosody processing in speech production: pre-evaluation of a fMRI study. Proceedings $14^{\text {th }}$ International Congress of Phonetic Sciences, San Francisco, 2339-2342.

Mayer, J., Wildgruber, D., Riecker, A., Dogil, G., Ackermann, H. \& Grodd, W. (2002). Prosody production and perception: Converging evidence from fMRI studies. Proceedings of Speech Prosody, Aix en Provence, France, 487-490..

Mertus, J. (1989). BLISS User’s Manual, Brown University, Providence, RI.

Nagel, H., Shapiro, L., \& Nawy, R. (1994). Prosody and the processing of filler-gap sentences. Journal of Psycholinguistic Research, 23, 473-485.

Nespor, M. \& Vogel, I. (1986). Prosodic phonology. Dordrecht: Foris.

Pell, M. \& Baum, S. (1997). The ability to perceive and comprehend intonation in linguistic and affective contexts by brain damaged adults. Brain and Language, 57, 80-99.

Poeppel, D. (2003). The analysis of speech in different temporal integration windows: 
cerebral lateralization as 'asymmetric sampling in time'. Speech Communication, 41, 245-255.

Price, P. J., Ostendorf, S., Shattuck-Hufnagel, S. \& Fong, C. (1991). Use of prosody in syntactic disambiguation. Journal of the Acoustic Society of America, 90, 29562970.

Ryalls, J. (1982). Intonation in Broca's aphasia. Neuropsychologia, 20, 355-360.

Schirmer, A. Alter, K., Kotz, S. A., and Friederici, A. D. (2001). Lateralization of prosody during language production: a lesion study. Brain and Language, 76, 117.

Selkirk, E. (1984). Phonology and syntax: The relation between sound and structure. Cambridge, MA: MIT Press.

Shapiro B. E., \& Danly, M. (1985). The role of the right hemisphere in the control of speech prosody in prepositional and affective contexts. Brain and Language, 25, 19-36.

Shapiro, L. \& Nagel, N. (1985). Lexical properties, prosody and syntax: Implications for normal and disordered language. Brain and Language, 50, 240-257.

Smith, B. L. (1980). Cortical stimulation and speech timing-A preliminary observation. Brain and Language, 10, 89-97.

Stirling, L. and Wales, R. (1996). Does prosody support or direct sentence processing. Language and Cognitive Processes, 11, 193-212.

Van Lancker, D. (1980). Cerebral lateralization of pitch cues in the linguistic signal. International Journal of Human Communication, 13, 227-277.

Van Lancker, D. (2004). When novel sentences spoken or heard for the first time in the 
history of the universe are not enough: toward a dual-process model of language. International Journal of Language \& Communication Disorders, 39, 1-44.

Van Lancker, D. \& Ohnesorge, C. (2002). Personally familiar proper names are relatively successfully processed in the human right hemisphere, or, the missing link. Brain \& Language, 77, 135-165.

Van Lancker, D. \& Sidtis, J. J. (1992). The identification of affective-prosodic stimuli by left- and right-hemisphere-damaged subjects: All errors are not created equal. Journal of Speech and Hearing Research, 35, 1963-970.

Walker, J. P., Fongemie, K., \& Daigle, T. (2001). Prosodic facilitation in the resolution of syntactic ambiguities in subjects with left and right hemisphere damage. Brain and Language , 78 , 169-196.

Walker, J.P., Pelletier, R., \& Reif, L. (2004). The production of linguistic structures in subjects with right hemisphere damage. Clinical Linguistics and Phonetics, 18, 85-106.

Warren, P. (1985). The temporal organization and perception of speech. Unpublished doctoral dissertation, University of Cambridge.

Weintraub, S., Mesulam, M., \& Kramer, L. (1981). Disturbances in prosody: A righthemisphere contribution to language. Archives of Neurology, 38, 742-744.

Wiig, E. H. \& Secord, W. A. (1989). Test of language competence- expanded edition. Harcourt, Brace and Jovanovich, San Antonio.

Wilson, B., Cockburn, J. \& Halligan, P. (1987). Behavioral Inattention Test. Thames Valley Test Company, Titchfield, UK.

Zatorre, R. J., Evans, A. Meyer, E. \& Gjedde, A. (1992). Lateralization of phonetic and 
Neural substrates 26

pitch discrimination in speech processing. Science, 256, 846-849. 


\section{Appendix 1: List of stimulus sentences and biasing contexts}

\section{Parenthetical-type Sentences}

1. Biasing Context: I can't remember exactly what I said to the mechanic Non-Integrated: I told him, I think, that the car battery is dead.

Biasing Context: I brought the car in for service and talked with the mechanic. Integrated: I told him I think that the car battery is dead.

2. Biasing Context: Your team will work well with you this term. Non-Integrated: They believe, you realize, your goals.

Biasing Context: They admire you and the way you get things done. Integrated: They believe you realize your goals.

3. Biasing Context: Ian is too disorganized to leave on time. Non-Integrated: Does Ian think, I wonder, when he will leave?

Biasing Context: Ian has not yet told me what flight he is on. Integrated: Does Ian think I wonder when he will leave?

4. Biasing Context: Mel asked if you could give him a ride to the party. Non-Integrated: Mel knew, by the way, you were driving.

Biasing Context: It was obvious you had been drinking. Integrated: Mel knew by the way you were driving.

5. Biasing Context: No one wants to have to fire a co-worker. Non-Integrated: The job, I mean, is never easy.

Biasing Context: I'm not talking about a job that's hard some times and easy others. Integrated: The job I mean is never easy.

6. Biasing Context: Jane is eager to attend my party but is unsure of her schedule. Non-Integrated: Jane hopes, I believe, that she's coming.

Biasing Context: Jane tried to convince me last week that she would make it to my party. Integrated: Jane hopes I believe that she’s coming

7. Biasing Context: I hadn’t heard what the defendant said, but Tom did. Non-Integrated: "I heard what", he said, "and it's true".

Biasing Context: I had read this honest report before and saw the author speak. Integrated: I heard what he said and it's true.

8. Biasing Context: Teaching chemistry is a very difficult task. Non-Integrated: Teaching a language, you understand, is much easier.

Biasing Context: I had a very hard time trying to teach Chinese 101 Integrated: Teaching a language you understand is much easier.

\section{Appositive-type Sentences}

1. Biasing Context: The Smiths didn't know what to do while their TV was broken.

Non-Integrated: The neighbors who always read, the Daleys, were amused.

Biasing Context: There was a funny cartoon in the local paper today. Integrated: The neighbors who always read the dailies were amused.

2. Biasing Context: These pictures are from a village where one group does the washing and another the wringing.

Non-Integrated: These are the ones who wring, the Belz. 
Biasing Context: Here is the list of people in the music group. Integrated: These are the ones who ring the bells.

3. Biasing Context: Most of the women had forgotten what happened. Non-Integrated: Only one remembered, the lady in red.

Biasing Context: Most of the people forgot about the strange woman. Integrated: Only one remembered the lady in red.

4. Biasing Context: I can’t remember whether you are going to Italy or France. Non-Integrated: Wherever you are, in Italy or France, write to me.

Biasing Context: I hope you enjoy your tours of Rome, Paris and the other cities in Italy and France. Integrated: Wherever you are in Italy or France, write to me.

5. Biasing Context: Each family has two sons, but in only one are they old enough to drive. Non-Integrated: Those are the brothers who drive, the Carrs.

Biasing Context: I want to show you which boys own those two matching Porsches. Integrated: Those are the brothers who drive the cars.

6. Biasing Context: Almost no one believed that bible story. Non-Integrated: Only one believed, the preacher from Georgia.

Biasing Context: Hardly anyone on the jury believed the minister's story. Integrated: Only one believed the preacher from Georgia.

7. Biasing Context: All but one family thought there was plenty of food the supper. Non-Integrated: The family that likes to eat, the Bergers, left hungry.

Biasing Context: Several families were invited to a vegetarian supper. Integrated: The family that likes to eat the burgers left hungry.

8. Biasing Context: One family in this picture is always moving around and another never does.

Non-Integrated: Those are the ones who roam, the Rhodes.

Biasing Context: One group of vagabonds in the picture is always on the move; the other lives underground.

Integrated: Those are the ones who roam the roads.

\section{Tag-type Sentences}

1. Biasing Context: Dave is always angry but he can't say why.

Non-Integrated: Dave will never know why he's enraged, will he?

Biasing Context: Dave insulted Willy without realizing it. Integrated: Dave will never know why he’s enraged Willy.

2. Biasing Context: After years of being banned, you could now sing that tune again. Non-Integrated: Miles didn't know the melody was allowed, did he?

Biasing Context: Miles thought the music box would play a sweet tune. Integrated: Miles didn't know the melody was a loud ditty.

3. Biasing Context: Ben stayed long after everyone else had left. Non-Integrated: Ben would never leave, would he?

Biasing Context: Ben stood guard at Woody’s bedside. Integrated: Ben would never leave Woody.

4. Biasing Context: My reply to the question about our religious beliefs was simple. 
Non-Integrated: Miriam and I don't believe, do we?

Biasing Context: Dewey's lies are old news to us.

Integrated: Miriam and I don't believe Dewey.

5. Biasing Context: George went out instead of studying for his exam.

Non-Integrated: George isn't worrying, is he?

Biasing Context: George's threats affect some people, but Izzy ignores them. Integrated: George isn't worrying Izzy.

6. Biasing Context: I want more information about where you're going.

Non-Integrated: You're not going to meet my friend Kim, are you?

Biasing Context: I won't let you meet my newest friend.

Integrated: You're not going to meet my friend Kim R. Yu.

7. Biasing Context: His train was due in, but I haven’t seen him.

Non-Integrated: He musn't have arrived at all, must he?

Biasing Context: Despite his time in the damp basement, he got home smelling fresh.

Integrated: He mustn't have arrived at all musty.

8. Biasing Context: I shall sing a high G, but I'm not sure about Bill.

Non-Integrated: He shall sing a C, shan't he?

Biasing Context: The old sailor stepped onto the stage.

Integrated: He shall sing a sea shanty. 


\section{Author Note}

This research was supported by a grant (MOP-11290) from the Canadian Institutes of Health Research (CIHR) to Shari Baum. Portions of this work were presented at the October 2004 meeting of the Academy of Aphasia, Chicago, IL.

The authors would like to gratefully acknowledge the assistance of Pablo Ruiz, Erin Vensel, Meg Grant and Lisa Trayhern.

This study was conducted while Amee P. Shah was at McGill University; since then, her affiliation and contact information have changed to Cleveland State University. 


\section{Table Captions}

Table 1. Background information on subjects

Table 2. Exemplar target sentences of three stimulus types with their biasing contexts

Table 3. Percent errors on the perceptual task for each speaker, collapsed across listeners 


\section{Figure Captions}

Figure 1 a-c: Mean pre-boundary word durations (proportions) in integrated and nonintegrated versions of three sentence types (a. Parentheticals, b. Appositives, c. Tags) for all speaker groups. Asterisks indicate statistically significant differences.

Figure 2 a-c. Mean pause durations (proportions) in integrated and non-integrated versions of three sentence types (a. Parentheticals, b. Appositives, c. Tags) for all speaker groups. Asterisks indicate statistically significant differences. Figure 3 a-c. Mean Pre-boundary/Post-boundary F0 ratios in integrated and non-integrated versions of three sentence types (a. Parentheticals, b. Appositives, c. Tags) for all speaker groups. Asterisks indicate statistically significant differences.

Figure 4. Percent errors on the perceptual task, collapsed across listeners and speakers 